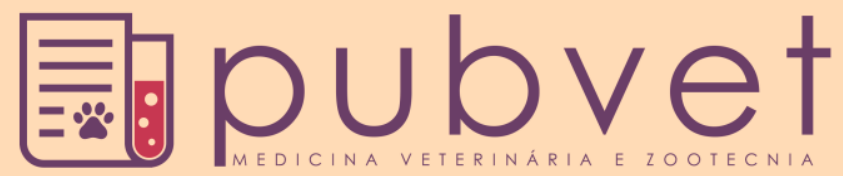

HTTP://DX.DOI.ORG/10.22256/PUBVET.V11N9.854-859

\title{
Avaliação in vitro do efeito acaricida do extrato etanólico das folhas de Hyptis suaveolens (L.) Poit (Lamiaceae) sobre fêmeas ingurgitadas de Rhipicephalus sanguineus (Latraille, 1806) (Acari:Ixodidae)
}

\author{
Daiane de Sousa Estrela ${ }^{1}$, Raylson Pereira de Oliveira ${ }^{2}$, Jeane Gomes de Matos ${ }^{1}$, Dulcilany \\ Pereira Cardoso ${ }^{3}$, Paulo Franklin Pereira de Sousa ${ }^{4}$, João Sammy Nery de Souza ${ }^{5}$, Pollyana \\ Oliveira da Silva ${ }^{6}$, Jamylla Mirck Guerra de Oliveira ${ }^{7}$, Márcia Paula Oliveira Farias ${ }^{7 *}$
}

\footnotetext{
${ }^{I}$ Graduando em Medicina Veterinária pela Universidade Federal do Piauí, Campus professora Cinobelina Elvas, Bom Jesus, Piauí, Brasil. E-mail: dayane-sousa-estrela@hotmail.com jeanegomesdematos@gmail.com

${ }^{2}$ Residente em Medicina Veterinária pela Universidade Federal Rural de Pernambuco, Campus Recife, Departamento de Medicina Veterinária, Recife, Pernambuco, Brasil. E-mail: raylson.oliveira@ hotmail.com.br

${ }^{3}$ Médica Veterinária autônoma, Bom Jesus, Piauí, Brasil. E-mail: dulcy_pc@ hotmail.com

${ }^{4}$ Graduando em Engenharia Florestal pela Universidade Federal do Piauí, Campus professora Cinobelina Elvas, Bom Jesus, Piauí, Brasil. E-mail: pauloflankin@gmail.com

${ }_{5}^{5}$ Docente da Universidade Federal do Piauí, Campus professora Cinobelina Elvas, Curso de Ciências Biológicas, Bom Jesus, Piauí, Brasil. sammynery@ufpi.edu.br

${ }^{6}$ Docente da Universidade Federal do Piauí, Campus professora Cinobelina Elvas, Curso de Zootecnia, Bom Jesus, Piauí, Brasil. E-mail: pollyanaodasilva@hotmail.com

${ }_{7}^{7}$ Docente da Universidade Federal do Piauí, Campus professora Cinobelina Elvas, Curso de Medicina Veterinária, Bom Jesus, Piauí, Brasil. Email:myllamirck@hotmail.com

*autor para correspondência marciapbo@gmail.com
}

RESUMO. O objetivo deste estudo foi avaliar "in vitro" o efeito acaricida do extrato etanólico das folhas de Hyptis suaveolens sobre fêmeas ingurgitadas de Rhipicephalus sanguineus. Foram coletadas manualmente, 150 fêmeas ingurgitadas de carrapatos, de cães naturalmente infestados, da microrregião de Bom Jesus-PI, Brasil. Para realização do teste de imersão sobre fêmeas ingurgitadas, foram testadas 3 diluições do extrato etanólico nas concentrações de 0.1 , 1.0 e $10.0 \%$, obtidas a partir do teste de toxicidade frente a Artemia salina. Foram utilizadas 10 fêmeas ingurgitadas para cada tratamento. Os testes foram realizados em triplicata. Para o controle negativo, foi utilizado água destilada e, para o controle positivo, Cypermetrina. Foi observado, nas concentrações testadas, eficácia de 29,93\%, 32,71\%, e 30,63\% respectivamente. Os dados obtidos nesse estudo revelaram que o extrato etanólico das folhas de $H$. suaveolens, nas concentrações testadas, não apresenta atividade acaricida para fêmeas de Rhipicephalus sanguineus, segundo padrões do Ministério da Agricultura.

Palavras chave: Carrapatos, controle, medicina veterinária preventiva

\section{In vitro evaluation of the acaricidal effect of ethanolic extract of Hyptis suaveolens (L.) Poit (Lamiaceae) leaves on engorged females of Rhipicephalus sanguineus (Latraille, 1806) (Acari: Ixodidae)}

ABSTRACT. The objective of this study was to evaluate the acaricidal effect in vitro of the ethanolic extract of Hyptis suaveolens leaves on engorged females of Rhipicephalus sanguineus. A total of 150 engorged females ticks were collected manually from dogs naturally infested in Bom Jesus-PI, Brazil. To perform the immersion test on engorged females, 3 
dilutions of the ethanolic extract were tested at concentrations of $0.1,1.0$ and $10.0 \%$, obtained from the toxicity test against Artemia salina. Ten engorged females were used for each treatment. The tests were performed in triplicate. For the negative control, distilled water was used and, for the positive control, was used Cypermethrin. Efficacy of 29.93\%, 32.71\%, and $30.63 \%$, respectively, were observed at the tested concentrations. The data obtained in this study revealed that the ethanolic extract of the leaves of $H$. suaveolens in the tested concentrations does not presents acaricidal activity for females of Rhipicephalus sanguineus, according to standards of the Ministry of Agriculture.

Keywords: Ticks, control, preventive veterinary medicine

\section{Evaluación in vitro del efecto acaricida del extracto de hojas de Hyptis suaveolens (L.) Poit (Lamiaceae) sobre las hembras ingurgitadas de Rhipicephalus sanguineus (Latraille, 1806) (Acari: Ixodidae)}

RESUMEN. El objetivo de este estudio fue evaluar "in vitro" el efecto acaricida del extracto
de las hojas de Hyptis suaveolens sobre Rhipicephalus sanguineus. Fueron colectadas
manualmente, 150 garrapatas (hembras ingurgitadas), de perros naturalmente infestados,
procedentes de la microrregión de Bom Jesus estado de Piauí, Brasil. Para la realizar la prueba
de inmersión sobre las hembras ingurgitadas, fueron testadas tres diluciones etanólicas en
concentraciones de $0.1,1.0$ y $10.0 \%$, obtenidas a partir del test de toxicidad frente a Artemia
salina. Se utilizaron 10 hembras ingurgitadas para cada tratamiento. Los test se realizaron en
triplicado. Para el control negativo, se utilizó agua destilada y para el control positivo,
Cypermetrina. Fue observado, en las concentraciones testadas, eficacia de $29,93 \%, 32,71 \%$, y
$30,63 \%$ respectivamente. Los datos obtenidos en este estudio revelan que el extracto de las
hojas H. suaveolens, en las concentraciones testadas, no presenta actividad acaricida para las
hembras Rhipicephalus sanguineus, teniendo en cuenta patrones del Ministerio de Agricultura.

Palabras clave: garrapatas, control, medicina veterinaria preventiva

\section{Introdução}

Rhipicephalus sanguineus (Latreille, 1806) é um carrapato da família Ixodidae, tem origem africana, sendo um dos principais ectoparasitos de cães. Apresenta ampla distribuição nos ambientes urbanos e rurais e é classificado como um dos principais vetores de patógenos para cães domésticos (Labruna, 2004).

Nas últimas décadas, tanto a prevalência quanto a intensidade de infestações por carrapatos em cães vêm aumentando em todo território brasileiro (Labruna, 2004). Muito embora haja vários relatos de parasitismo por $R$. sanguineus em várias espécies animal em diferentes regiões geográficas, o cão doméstico, sem dúvida, é o principal hospedeiro para todos os estágios parasitários desse carrapato (Walker et al., 2005).

Eventualmente esse parasita pode ser visto parasitando o homem devido à sua estreita ligação com o cão. Contudo, esse carrapato tornou-se de grande importância para a saúde pública, pois embora seja um parasitismo incomum, tem a capacidade de transmitir uma série de agentes patogênicos para o homem (Dantas-Torres et al., 2006).

Atualmente, a resistência parasitária pelo uso indiscriminado de substâncias carrapaticidas vem gerando graves prejuízos a criadores de cães e até mesmo médicos veterinários pelo uso incorreto desses carrapaticidas, além de contaminações ambientais e de risco à saúde animal e humana. Esses prejuízos se estendem ao tratamento e controle de enfermidades que tem o $R$. sanguineus como vetor de hemoparasitas como a Ehrlichia canis, Babesia canis, Haemobartonella canis, Hepatozoon canis e Anaplasma platys (Paz et al., $\underline{2008}$, Farias et al., 2009). No Brasil, não há relatos de resistência a grupos farmacológicos por $R$. sanguíneus, mas com o uso indiscriminado desses, 
possivelmente a resistência pode já estar instalada, ou futuramente, fazendo necessário testes com novas drogas, com finalidade de identificar grupos farmacológicos aos quais os carrapatos sejam sensíveis, e seguindo esse contexto vem sendo realizado testes com princípios ativos de plantas, para o controle desse ectoparasita (Chagas, 2004).

A fitoterapia, que é um ramo da farmacologia que tem como base estudos de princípios ativos de plantas, vem sendo uma alternativa de baixo custo para o controle desses parasitos, tendo em vista a grande biodiversidade brasileira (Rezende and Cocco, 2002).

Diante do exposto, esse trabalho foi desenvolvido com objetivo de avaliar "in vitro" a atividade acaricida do extrato etanólico das folhas de Hyptis suaveolens sobre fêmeas ingurgitadas de $R$. sanguineus.

\section{Material e Métodos}

\section{Coleta de material e preparo do extrato}

As folhas de $H$. suaveolens foram coletadas nas margens da rodovia que liga o município de Bom Jesus Piauí ao Campus Professora Cinobelina Elvas (CPCE), e levadas posteriormente ao laboratório de química orgânica. A exsicata da planta foi identificada e armazenada no "Herbário Graziela Barroso" - TEPB/UFPI, com registro de $\mathrm{n}^{\circ} 28.826$. No laboratório, as folhas foram secas por 8 dias em estufa com temperatura de $37^{\circ}$, e circulação de ar. Em seguida foram trituradas em moinho de facas tipo Willye, modelo SL-032, e submetidas à extração a frio com etanol absoluto $99,5 \%$ por $72 \mathrm{~h}$. A solução foi agitada diariamente durante o período de extração. $\mathrm{O}$ extrato foi filtrado em papel de filtro e destilado à temperatura de $60^{\circ} \mathrm{C}$, sob pressão reduzida de 500 a $750 \mathrm{mmHg}$, em evaporador rotativo, modelo 802. O material concentrado foi liofilizado para o uso.

\section{Bioensaio de toxicidade frente à Artemia salina}

Para a obtenção e escolha das concentrações a serem utilizadas no teste de eficiência acaricida foi realizado um bioensaio preliminar de toxicidade frente à A. salina como descrito por Meyer et al. (1982) com algumas adaptações. Com o auxílio de uma pipeta de Pasteur foram transferidos 10 náuplios do microcrustáceo para cada tubo de ensaio e o volume foi então completado para $9,0 \mathrm{ml} \mathrm{da}$ solução salina previamente preparada (água do mar artificial). Foi adicionado, em seguida, 1,0 $\mathrm{ml}$ do extrato etanólico das folhas de $H$. suaveolens em diferentes concentrações $(0,1 ; 1,0 ; 10,0 ; 100,0$ e $1000,0 \mu \mathrm{g} / \mathrm{ml})$. O teste foi realizado em triplicata para cada concentração do extrato etanólico de Hyptis suaveolens. Para o controle negativo foi adicionado apenas 10 náuplios em 10,0 ml de água marinha artificial, também em triplicata, totalizando 18 tubos no teste. Após 24 horas da realização do teste, foi realizada a observação com o auxílio de uma lupa, sendo considerados náuplios mortos todos que não apresentavam qualquer movimento ativo em cerca de vinte segundos de observação.

\section{Teste de eficácia acaricida "in vitro"}

Para avaliação "in vitro" da atividade acaricida do extrato etanólico das folhas de $H$. suaveolens, foram coletadas manualmente 150 fêmeas ingurgitadas do carrapato da espécie $R$. sanguineus, provenientes de cães naturalmente infestados, os quais, a mais de 90 dias, não passavam por tratamento acaricida, sendo esses cães pertencentes à microrregião da cidade de Bom Jesus, Piauí. Após coleta, os carrapatos foram levados ao Laboratório de Doenças Parasitárias dos Animais Domésticos da Universidade Federal do Piauí, Campus Professora Cinobelina Elvas, em recipientes plásticos sob aeração com o auxílio de aerador para aquários. As fêmeas foram limpas com papel absorvente, pesadas e colocadas em placas de Petri, divididas em grupos de 10. Os testes foram realizados em triplicada, totalizando 30 fêmeas por grupo. Foram preparadas três diluições do extrato etanólico das folhas de $H$. suaveolens, utilizando concentrações obtidas após teste de toxicidade frente à $A$. salina. As concentrações do extrato utilizadas foram: $0,1 \%$ (grupo 1), 1,0\% (grupo 2) e 10\% (grupo 3) devido a toxidade encontrada no teste com A. salina ser bem semelhante entre essas concentrações, além do controle positivo (CP) em que foi utilizado um medicamento de uso veterinário, a Cypermetrina, e controle negativo $(\mathrm{CN})$, utilizando água destilada. Em seguida as fêmeas foram submetidas ao teste de imersão de acordo com a metodologia proposta por Drummond et al. (1973).

Utilizou-se no momento do teste, copos plásticos descartáveis de $50 \mathrm{ml}$ contendo $10 \mathrm{ml}$ de cada concentração do extrato, bem como dos controles utilizados, mantendo-se o liquido em constante 
agitação durante 5 minutos. Após o banho de imersão, o excesso das soluções foi retirado usandose papel absorvente, e as fêmeas de cada grupo testado foram colocadas em placas de Petri em temperatura ambiente para postura.

Dez dias após o início de postura das fêmeas, procedeu-se a pesagem de massa de ovos em balança analítica, e transferência desses ovos para seringas plásticas de $20 \mathrm{ml}$, todas identificadas e vedadas com algodão hidrofóbico para eclosão das larvas. Após 20 dias, foi realizada a contagem descritiva para avaliação da quantidade de eclosão, onde foi estimado objetivamente, adotando-se a verificação visual com intervalos de $10 \%$ como parâmetro.

O cálculo da eficácia do produto foi realizado usando-se as fórmulas matemáticas abaixo descritas (Drummond et al., 1973).

$\mathrm{RE}=$ Reprodução estimada

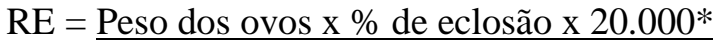

Peso das fêmeas

$\mathrm{EP}=$ Eficácia do produto

${ }^{*}$ Constante que indica o número de ovos presentes em $1 \mathrm{~g}$ de postura

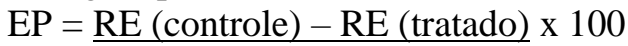

$$
\mathrm{RE} \text { (controle) }
$$

Os dados obtidos foram interpretados de acordo com a legislação pertinente à comercialização de carrapaticidas no Brasil e os resultados foram interpretados considerando-se como eficácia acaricida o valor mínimo de $95 \%$.

\section{Analise estatística}

Os dados foram tabulados e a quantidade de náuplios mortos de acordo com as concentrações do extrato etanólico de $H$. suaveolens (L) Poit (Lamiaceae) foi determinada estatisticamente por meio de análise de variância (ANOVA) pelo programa estatístico Statistical Analysis System (SAS), versão 9.1 (SAS, 2004), a comparação entre as médias foi realizada pelo teste Tukey considerando como significativo quando $p \leq 0,05$.

\section{Resultados e Discussão}

O extrato etanólico das folhas de Hyptis suaveolens apresentou toxicidade frente à Artemia salina em todas as concentrações testadas, sendo identificada uma alta toxicidade para as concentrações 100 e $1000(\mu \mathrm{g} / \mathrm{ml})$, nas porcentagens de 93,5 e $100 \%$ respectivamente. Em relação às concentrações 0,$1 ; 1,0$ e 10,0 também não houve uma alta variação entre as medias de náuplios mortos, observando as porcentagens das médias da concentração $0,1(\mu \mathrm{g} / \mathrm{ml})$, apresentou toxicidade de $50,2 \%$, a concentração $1,0(\mu \mathrm{g} / \mathrm{ml}), 52,25 \%$ e a concentração de $10,0(\mu \mathrm{g} / \mathrm{ml})$, foi equivalente a $58,50 \%$ (Tabela 1 ).

Tabela 1. Mortalidade média de náuplios de Artemia salina de acordo com as concentrações do extrato etanólico das folhas de Hyptis suaveolens

\begin{tabular}{lcc}
\hline $\begin{array}{l}\text { Concentração do extrato } \\
\text { etanólico de } H \text {. suaveolens, } \\
\mu \mathrm{g} / \mathrm{ml}\end{array}$ & $\begin{array}{c}\text { Média de náuplios } \\
\text { mortos, } \%\end{array}$ & $\begin{array}{c}\text { Desvio } \\
\text { Padrão }\end{array}$ \\
\hline 0 (controle) & $23.10^{\mathrm{c}}$ & $\pm 6,92$ \\
0,1 & $50.27^{\mathrm{b}}$ & $\pm 10,20$ \\
1,0 & $52.36^{\mathrm{b}}$ & $\pm 4,64$ \\
10,0 & $58.50^{\mathrm{b}}$ & $\pm 8,60$ \\
100,0 & $93.47^{\mathrm{a}}$ & $\pm 6,27$ \\
1000,0 & $100.00^{\mathrm{a}}$ & 0 \\
\hline A
\end{tabular}

As médias seguidas pela mesma letra não diferem estatisticamente entre si (ANOVA One-Way, seguida pelo Teste de Tukey ao nível considerado como significativo quando $\mathrm{p} \leq 0,05$ ).

Observou-se que o extrato etanólico das folhas e $H$. suaveolens não apresentou atividade acaricida frente às fêmeas ingurgitadas de $R$. sanguineus (Tabela 2).

Tabela 2. Eficácia acaricida do extrato etanólico das folhas e Hyptis suaveolens frente às fêmeas ingurgitadas de Rhipicephalus sanguineus (Latraille, 1806)

\begin{tabular}{lcccc}
\hline Concentração & $\begin{array}{c}\text { Número de } \\
\text { teleóginas }\end{array}$ & $\begin{array}{c}\text { Eclosão das } \\
\text { larvas, \% }\end{array}$ & RIE & EP, \% \\
\hline $\mathrm{CN}$ & 30 & $100 \%$ & 144,16 & - \\
$\mathrm{CP}$ & 30 & 98,33 & 46,3 & 67,88 \\
$0,1 \%$ & 30 & 100 & 100 & 29,93 \\
$1,0 \%$ & 30 & 96,6 & 97 & 32,71 \\
$10, \%$ & 30 & 96,6 & 100 & 30,63 \\
\hline CN - Água destilada (controle negativo); CP - Controle positivo; RIE \\
- índice de eficiência reprodutiva; EP - eficácia do produto.
\end{tabular}

As fêmeas ingurgitadas de $R$. sanguineus submetidas ao banho de imersão na concentração $0,1 \%$ do extrato apresentaram o maior índice de eficiência reprodutiva e uma alta porcentagem de eclosão das larvas chegando a 100\%, sendo observado uma menor eficácia do produto nessa concentração. Quando comparado a estudos como 
de Fernandes et al. (2010), em que os mesmos utilizaram óleo essencial do NIM (Azadirachta indica) sobre fêmeas ingurgitadas de $R$. sanguineus, na menor concentração do seu estudo (2\%) a eficiência do produto foi menor em relação a concentração de $0,1 \%$ do extrato etanólico de $H$. suaveolens, sendo esse um bom indicativo para futuros testes com o óleo essencial da mesma.

A concentração $1,0 \%$ apresentou a maior eficácia acaricida em relação às outras concentrações, chegando a $32,7 \%$, sendo superior a concentração de $10 \%$, pois o que diferiu no teste de eficiência acaricida foram só as concentrações testadas, o mesmo pode ser observado no estudo de Andrade et al. (2013), em relação aos extrato obtido de Capim Santo nas concentrações de $2,5 \%, 5,0 \%$ e $10 \%$, que apresentaram eficácia de 90,0\%, 43,5\%, e 72,1\%, respectivamente, em que a menor concentração $(2,5 \%)$ no referido estudo apresentou maior efeito acaricida sobre fêmeas ingurgitadas de $R$. sanguineus. Foi possível observar no presente estudo que o controle positivo (Cypemetrina) obteve uma baixa eficácia do produto chegando a 67,9\%, já apresentando uma resistência do $R$. sanguineus ao uso do medicamento. A organização mundial para alimentos e agricultura, preconiza como $80 \%$ de eficácia um excelente resultado.

As plantas da família Lamiaceae são de grande importância econômica graças ao seu alto teor de óleos essenciais aromáticos, sendo que $H$. suaveolens, pertencente a essa família, apresenta em sua composição química, compostos com atividade biológica já comprovada (Falcão and Menezes, 2003).

Moreira et al. (2010) relatam o uso do óleo extraídos das folhas de H. suaveolens, que revelou uma forte atividade antifúngica, inibindo $\mathrm{o}$ crescimento de alguns Aspergillus, o que demonstrar que este tem um potencial terapêutico.

O fato do extrato etanólico das folhas de $H$. suaveolens apresentar baixa eficácia carrapaticida e alta atividade fungicida pode ser explicada devido à o mecanismo de ação de algumas substancias presente no extrato, onde essas substancias só seriam ativadas com a ingestão da mesma pelos carrapatos o que tornaria a metodologia inadequada para a avaliação da atividade acaricida frente às fêmeas ingurgitadas (Castro et al., 2011).

\section{Conclusão}

O extrato etanólico das folhas de $H$. suaveolens sobre fêmeas ingurgitadas de $R$. sanguineus não apresentou atividade acaricida satisfatória nas concentrações testadas, um posterior estudo pode ser realizado usando concentrações superiores em relação ao presente estudo. Tendo este estudo relevância na pesquisa de fitoterápicos com ação frente ao $R$. sanguineus, já que existe poucos relatos na literatura sobre o uso de fitoterápicos no controle do mesmo.

\section{Referências Bibliográficas}

Andrade, A. W. F., Soares, G. W. N., Bezerra, T. I. C., Souza, M. G., Silva, F. M. F. M., Almeida, J. R. G. S. \& Horta, M. C. 2013. Avaliação da eficácia de extratos vegetais no controle do Rhipicephalus sanguineus provenientes do município de Juazeiro, Bahia. Evolvere Scientia, 2, 1-8.

Castro, K. N. C., Vasconcelos, L., Costa, J. V., Santos, R. C., Andrade, I. M. \& Ribeiro, V. Q. 2011. Extratos vegetais no controle in vitro do carrapato dos bovinos. Embrapa Meio-NorteArtigo em anais de congresso (ALICE). Cadernos de Agroecologia, Cruz Alta, Rio Grande do Sul.

Chagas, A. C. S. 2004. Controle de parasitas utilizanto extratos vegetais. Revista Brasileira de Parasitologia Veterináia, 13, 156-160.

Dantas-Torres, F., Figueredo, L. A. \& BrandãoFilho, S. P. 2006. Rhipicephalus sanguineus (Acari: Ixodidae), the brown dog tick, parasitizing humans in Brazil. Revista da Sociedade Brasileira de Medicina Tropical, 39, 64-67.

Drummond, R. O., Ernst, S. E., Trevino, J. L., Gladney, W. J. \& Graham, O. H. 1973. Boophilus annulatus and B. microplus: Laboratory Tests of Insecticides. Journal of Economic Entomology, 66, 130-133.

Falcão, D. Q. \& Menezes, F. S. 2003. Revisão etnofarmacológica, farmacológica e química do gênero Hyptis. Revista Brasileira de Farmácia, 84, 69-74.

Farias, M. P. O., Arruda, A. C., Faustino, M. A. G., Teixeira, W. C., Sousa, D. P., Wanderley, A. G. \& Alves, L. C. 2009. Potencial acaricida do óleo de andiroba Carapa guianensis Aubl. sobre 
fêmeas adultas ingurgitadas de Anocentor nitens Neumann, 1897 e Rhipicephalus sanguineus Latreille, 1806. Arquivo Brasileiro de Medicina Veterinária e Zootecnia, 61, 877-882.

Fernandes, J. I., Correia, T. R., Ribeiro, F. A., Cid, Y. P., Tavares, P. V. \& Scott, F. B. 2010. Eficácia in vitro do nim (Azadirachta indica) no controle de Rhipicephalus sanguineus (Latreille, 1806)(Acari: Ixodidae). Revista Brasileira de Medicina Veterinária, 32, 64-68.

Labruna, M. B. 2004. Biologia-ecologia de Rhipicephalus sanguineus (Acari: ixodidae). Revista Brasileira de Parasitologia $e$ Veterinária, 13, 123-124.

Meyer, B. N., Ferrigni, N. R., Putnam, J. E., Jacobsen, L. B., Nichols, D. E. j. \& McLaughlin, J. L. 1982. Brine shrimp: a convenient general bioassay for active plant constituents. Planta Médica, 45, 31-34.

Moreira, A. C. P., Lima, E. O., Wanderley, P. A., Carmo, E. S. \& Souza, E. L. 2010. Chemical composition and antifungal activity of Hyptis suaveolens (L.) poit leaves essential oil against Aspergillus species. Brazilian Journal of Microbiology, 41, 28-33.
Paz, G. F., Leite, R. C. \& Oliveira, P. R. 2008. Controle de Rhipicephalus sanguineus (Latreille, 1806)(Acari: Ixodidae) no canil da escola de veterinária da UFMG, Belo Horizonte, Minas Gerais, Brasil. Revista Brasileira de Parasitologia e Veterinária, 17, 41-44.

Rezende, H. A. \& Cocco, M. I. M. 2002. A utilização de fitoterapia no cotidiano de uma população rural. Revista da Escola de Enfermagem da USP, 36, 282-288.

SAS. 2004. SAS/STAT User guide, Version 9.1.2. SAS Institute Inc, Cary, NC, USA.

Walker, J. B., Keirans, J. E. \& Horak, I. G. 2005. The genus Rhipicephalus (Acari, Ixodidae): a guide to the brown ticks of the world. Cambridge University Press, London.

\section{Article History:}

Received 14 April 2017

Accepted 16 May 2017

Available on line 1 August 2017

License information: This is an open-access article distributed under the terms of the Creative Commons Attribution License 4.0, which permits unrestricted use, distribution, and reproduction in any medium, provided the original work is properly cited. 